\title{
Comparison between Intramedullary Nailing and Minimally Invasive Percutaneous Plate Osteosynthesis in Treatment of Humeral Shaft Fractures
}

\author{
Hang Yuan1, Ran Wang2, Jie Zheng2 and Yonghong Yang1,2 \\ IDepartment of Orthopedics, Zhejiang Hospital, Hangzhou, Zhejiang Province, China \\ ${ }^{2}$ Department of Orthopedics, People's Liberation Army 903 Hospital, Hangzhou, Zhejiang Province, China
}

\begin{abstract}
Objective: To compare the efficacy of intramedullary nailing (IMN) and minimally invasive percutaneous plate osteosynthesis (MIPO) in treatment of humeral shaft fractures.

Study Design: Descriptive study.

Place and Duration of Study: Department of Orthopedics, Zhejiang Hospital, China, during March 2010 to December 2016. Methodology: The study included patients with humeral shaft fractures who received surgery by IMN or MIPO. The intraoperative outcomes including operation time, bleeding volume, and the postoperative outcomes were also recorded. The Constant-Murley scores were used for assessment of function of shoulder joint and Mayo score was used for measurement of elbow joint function.

Results: Among a total of 436 patients with humeral shaft fractures, 204 cases received MIPO and 232 cases received IMN. The follow-up duration ranged from 16 36 months with a mean duration of $25.8 \pm 6.1$ months. The mean operation time, mean bleeding volume, mean hospitalisation time and mean healing time showed no significant difference between MIPO and IMN groups. No significant difference was observed in Mayo scores, but the Constant-Murley scores of MIPO group was significantly higher that the IMN group. Fracture nonunion was observed for $15(6.5 \%)$ cases in IMN group; however, only $1(0.5 \%)$ case was found to be nonunion in MIPO group. Meanwhile, $9(4.4 \%)$ cases showed radial nerve injury in the MIPO group, with no significant difference compared to the IMN group of $15(6.5 \%)$ cases.

Conclusion: Both the two methods were effective; however, MIPO method might be better for recovery of shoulder function and might reduce the nonunion rate.
\end{abstract}

Key Words: intramedullary nailing, invasive plate osteosynthesis, humeral fractures.

How to cite this article: Yuan $\mathrm{H}$, Wang R, Zheng J, Yang Y. Comparison between intramedullary nailing and minimally invasive percutaneous plate osteosynthesis in treatment of humeral shaft sractures. J Coll Physicians Surg Pak 2019; 29(10):942-5.

\section{INTRODUCTION}

Humeral shaft fractures account for about $20 \%$ of humeral fractures and $1-3 \%$ of all fractures.1,2 Generally, nonoperative treatment is suitable for most humeral shaft fractures. However, when cases are with open fractures, polytrauma or the non-operative treatment cannot achieve satisfied outcomes, the operative methods are needed. 3,4

Both intramedullary nailing (IMN) and the minimally invasive plate osteosynthesis (MIPO) technique are now widely adopted in surgical treatment of humeral shaft fractures. ${ }^{5}$ However, the controversy for which is better continues. Generally, the MIPO technique uses an indirect reduction technique with smaller incisions and less surgical injury than traditional plate osteosynthesis. ${ }^{6}$

Correspondence to: Yonghong Yang, Department of Orthopedics, Zhejiang Hospital, Hangzhou, Zhejiang Province, 310000, China E-mail:hong0178@outlook.com

Received: January 22, 2019; Revised: May 27, 2019;

Accepted: July 12, 2019
However, IMN also uses similar technique. ${ }^{7}$ On the other hand, some studies reported in distal extra-articular fractures, the reduction and stable fixation using IMN might be technically challenging. ${ }^{8}$

Compared with other common shaft fractures like tibial shaft fractures ${ }^{9}$, the comparison for efficacy between IMN and MIPO in treatment of humeral shaft fractures is still inadequate, and most comparison study included limited cases. Thus, studies with bigger study size are still needed to further confirm the efficacy of IMN and MIPO in treatment of humeral shaft fractures. This study aimed to compare the efficacy of MIPO and IMN in treatment of humeral shaft fractures and might provide more clinical evidence for the two methods.

\section{METHODOLOGY}

The present retrospective study included a total of 436 patients with humeral shaft fractures who went to the Department of Orthopeadic Surgery in Zhejiang Hospital and received surgery by IMN or MIPO during March 2010 to December 2016. The fractures were confirmed 


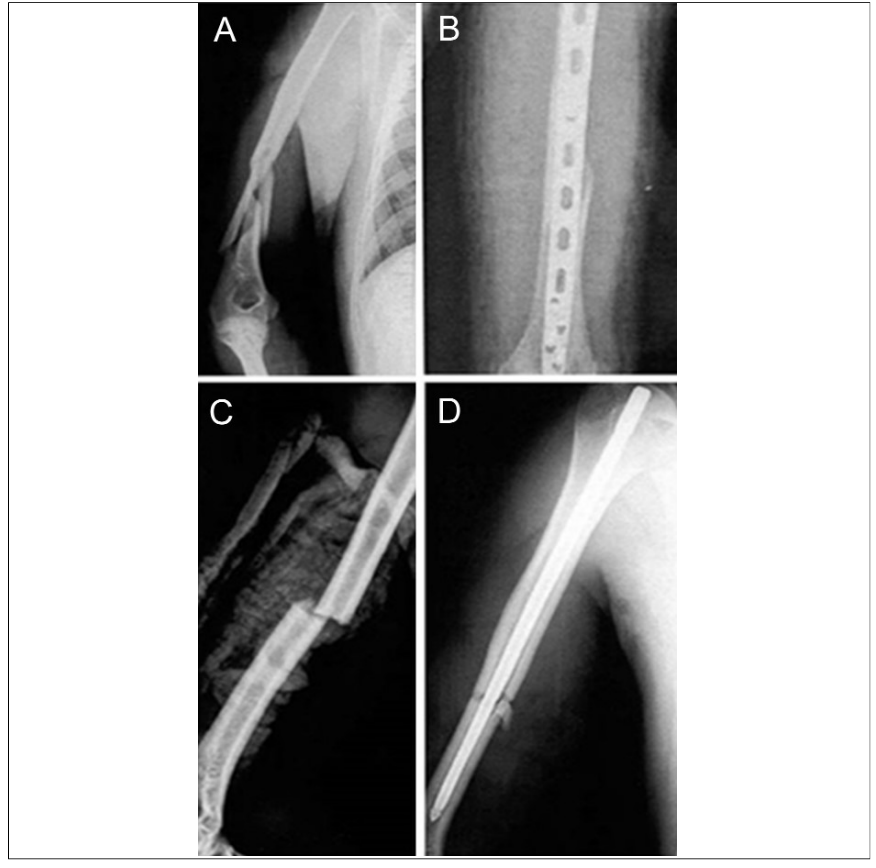

Figure 1: X-ray for patients with MIPO or IMN. (A) a patient with MIPO before the surgery; (B) the patient with MIPO after surgery; $(\mathbf{C})$ a patient with IMN before the surgery; (D) the patient with IMN after surgery.

by X-ray or CT scan (Figure 1). The patients received surgery by IMN or MIPO according to different case conditions and the surgeon's experience. The humeral shaft fracture was defined as fractures at the position between the surgical neck of humerus (more than $3 \mathrm{~cm}$ from surgical neck of humerus) and the olecranon fossa (about $5 \mathrm{~cm}$ from olecranon fossa). In all cases, the closed reduction could not achieve or did not give good outcomes. All included cases were unilateral fracture. Patients with history of humeral shaft fractures, with pathological fractures caused by tumor and less than 18 years of age were excluded. The MIPO surgery, as well as the IMN surgery, was conducted according to a consistent protocol. For MIPO, the locking compression plate (LCP, Weigao Group Medical Polymer Products Co., Ltd., Shandong, China) was used as reported previously. ${ }^{10}$ For IMN group, the surgery was conducted using interlocking intramedullary nailing as described elsewhere.11 Informed consent was obtained from all patients. The present study was approved by Ethics Committee of Zhejiang Hospital.

Patients' clinical characteristics such as age, gender, causes of fractures, and AO type were collected. The intraoperative outcomes including operation time, bleeding volume, and the postoperative outcomes, such as hospitalisation time, healing time and postoperative complications were also recorded. The Constant-Murley scores were used for assessment of function of shoulder joint; and Mayo score was used for measurement of elbow joint function. The follow-up for patients ranged from 16 36 months. The data of complications were collected during the follow-up period for each patient.
The data was expressed by mean $\pm S D$. Chi-square analysis was used for comparison of rates. Comparisons were conducted using student t-test when comparing two groups. It was considered to be statistically significant when $p$-value was less than 0.05 . All calculations were made using SPSS 18.0.

\section{RESULTS}

The present retrospective study included a total of 436 patients with humeral shaft fractures, with mean age of $41.2 \pm 13.9$ years. Among all patients, 242 cases were with AO A type, 143 cases were with AO B type and 61 cases were with $\mathrm{AO} C$ type. Follow-up duration ranged from 16 36 months with a mean duration of $25.8 \pm 6.1$. No significant difference in basic characteristics was found between the two groups (Table I).

The mean operation time, mean bleeding volume, mean hospitalisation time and mean healing time were compared between MIPO and IMN groups. As shown in Table II, all indices showed no significant difference between the two groups, suggesting that there was no significant difference for intraoperative outcomes and healing duration for the two methods.

To further compare the MIPO and IMN methods, Constant-Murley scores and Mayo scores for all patients

Table I: Basic clinical characteristics for patients with IMN and MIPO.

\begin{tabular}{|c|c|c|c|}
\hline Variables & MIPO, n=204 & IMN, n=232 & p-value \\
\hline Age, year & $41.5 \pm 14.6$ & $40.9 \pm 13.2$ & 0.662 \\
\hline Gender, female n (\%) & $85(41.7)$ & $104(44.8)$ & 0.658 \\
\hline Fracture side, $\mathrm{n}(\%)$ & & & 0.723 \\
\hline Left & 95 (46.6) & $114(49.1)$ & \\
\hline Right & $109(53.4)$ & $118(50.9)$ & \\
\hline AO type, n (\%) & & & 0.914 \\
\hline A & $115(56.4)$ & $127(54.7)$ & \\
\hline$B$ & $64(31.4)$ & $79(34.1)$ & \\
\hline $\mathrm{C}$ & $25(12.2)$ & $26(11.2)$ & \\
\hline Causes of fractures, $\mathrm{n}(\%)$ & & & 0.180 \\
\hline Traffic accident & $131(64.2)$ & $149(64.2)$ & \\
\hline Mechanical injury & $52(25.5)$ & $56(24.1)$ & \\
\hline Full & $20(9.8)$ & $26(11.2)$ & \\
\hline Other & $3(1.5)$ & $2(8.6)$ & \\
\hline Comorbidities, n (\%) & & & 0.865 \\
\hline Pelvic fracture & $37(18.1)$ & $49(21.1)$ & \\
\hline Fracture of tibia and fibula & $29(14.2)$ & $38(16.4)$ & \\
\hline Fracture of radius & $21(10.3)$ & $19(8.2)$ & \\
\hline Lumbar fracture & $11(5.4)$ & $17(7.3)$ & \\
\hline Flexor nerve injury & $10(4.9)$ & $7(3.0)$ & \\
\hline Follow-up, month & $25.7 \pm 6.1$ & $25.8 \pm 6.1$ & 0.851 \\
\hline
\end{tabular}

Comparison for operation time, bleeding volume, hospitalisation time and healing time between the two groups.

Table II: Comparison for operation time, bleeding volume, hospitalisation time and healing time between IMN and MIPO.

\begin{tabular}{l|c|c|c}
\hline Variables & MIPO, $\mathrm{n}=204$ & $\mathrm{IMN}, \mathrm{n}=232$ & $\mathrm{p}$-value \\
\hline Mean operation time (minutes) & $100.6 \pm 11.2$ & $99.6 \pm 21.1$ & 0.561 \\
\hline Mean bleeding volume (ml) & $97.7 \pm 24.0$ & $94.1 \pm 27.3$ & 0.155 \\
\hline Mean hospitalisation time (days) & $7.1 \pm 1.3$ & $6.9 \pm 1.2$ & 0.227 \\
\hline Mean healing time (days) & $12.1 \pm 1.4$ & $11.9 \pm 1.4$ & 0.147 \\
\hline
\end{tabular}


were recorded and compared three months after surgery for shoulder and elbow function, respectively. Results showed no significant difference in Mayo scores (90.6 \pm 3.5 for MIPO group and $89.8 \pm 5.5$ for IMN group, $p=0.110$ ), but the Constant-Murley scores of MIPO group $(90.3 \pm 3.5)$ was significantly higher than the IMN group $(82.1 \pm 5.5>p<0.001)$. This result indicated MIPO might be better for recovery of shoulder function of the humeral shaft fracture patients.

Fracture nonunion was observed for $15(6.5 \%)$ cases in IMN group, however only one $(0.5 \%)$ case was found to be nonunion in MIPO group $(p=0.022)$. Meanwhile, nine $(4.4 \%)$ cases showed radial nerve injury in the MIPO group, with no significant difference compared to the IMN group of $17(7.3 \%)$ cases $(p=0.382)$. These results suggested the fracture nonunion rate of MIPO might be lower than the IMN method.

\section{DISCUSSION}

Both MIPO and IMN now have been widely used in treatment of fractures of long shaft. However, the difference for the two methods in treatment of humeral shaft fractures is rarely reported. In the present study, both the two methods were effective; however, MIPO method might be better for recovery of shoulder function and might reduce the nonunion rate using a retrospective study.

MIPO has been reported in treatment of many kinds of long shaft fractures. Saengsin et al. showed the sonography assisted MIPO was effective in femoral shaft fracture and MIPO was as accurately as the radiography. 12 Recently, Zhang et al. showed MIPO could be used in treatment of displaced midshaft clavicular fracture, and MIPO technique with locking reconstruction plate was a feasible and worthwhile alternative method. ${ }^{13}$ It was also demonstrated in many cases that MIPO has efficacy with less complications in treatment of tibial shaft fracture.14,15 In the present study, we also demonstrated MIPO was effective in treatment of humeral shaft fractures, with very low rates of nonunion and nerve injury.

There are also many studies oppositional to IMN in treatment of long shaft fractures. Vallier et al. compared IMN with traditional plate and found although both methods were effective for treatment of distal tibia shaft fractures, the nonunion rate was higher for IMN. ${ }^{16}$ Vinzenz et al. studied 60 cases and recommended that elastic stable intramedullary nailing was the best for midshaft clavicular fractures without comminution. ${ }^{17}$ Jia et al. demonstrated a meta-analysis to compare IMN with plate technique and found both the methods were effective in treatment of humeral shaft fractures. However, nailing significantly increased the risk of shoulder complications. ${ }^{18}$ In the present study, also it was found that IMN could give good clinical outcomes with low complication rate in treatment of humeral shaft fractures. Similarly, in our research, we found that IMN and MIPO provided similar postoperative results, except for shoulder function and the complication of nonunion.

The comparison of MIPO and IMN can also be seen in several researches. Polat et al. indicated both IMN and MIPO had similar therapeutic efficacy regarding functional outcomes and none had a major advantage over the other in treatment of extra-articular distal tibial shaft fractures. 9 In a case-match controlled retrospective study for 30 cases, the authors reported MIPO was better than IMN with significant lower complication rate of nonunion and radial nerve injury. ${ }^{19}$ In a comparison for MIPO, IMN, and open reduction plate osteosynthesis for a total of 112 cases, it was also concluded that MIPO was overall better with respect to non-union, functional outcome and complications rate in treatment of humeral shaft fractures. 20 In this research, it was demonstrated that MIPO method might be better for recovery of shoulder function and might reduce the nonunion rate. However, the intraoperative outcomes and recovery time of the patients did not show difference. Thus, it was thought that both the two methods are acceptable in treatment of humeral shaft fractures.

The present study also has some limitations. First, this is a retrospective study, so a prospective study in the future may give deeper understanding. Secondly, the present study contains a medium sample size; more cases or meta-analysis including bigger size of patients may be needed.

\section{CONCLUSION}

Both the two methods were effective; however, MIPO method might be better for recovery of shoulder function and might reduce the nonunion rate. This study might give more clinical evidence for both MIPO and MIN in the treatment of humeral shaft fractures.

\section{FUNDING:}

This research was funded by Nanjing Military Region Key Project (No. 15ZD047).

\section{ETHICAL APPROVAL:}

The present study was approved by Ethics Committee of Zhejiang Hospital.

\section{PATIENTS' CONSENT:}

Informed consents were obtained from patients to publish the data concerning this case.

\section{CONFLICT OF INTEREST:}

Authors declared no conflict of interest.

\section{AUTHORS' CONTRIBUTION:}

HY: Written the manuscript. 
RW, JZ, YY: Discussion and comments on an earlier version of the manuscript. Approved the final manuscript.

\section{REFERENCES}

1. Mahabier KC, Vogels LMM, Punt BJ, Roukema GR, Patka P, Lieshout EMMV. Humeral shaft fractures: Retrospective results of non-operative and operative treatment of 186 patients. Injury 2013; 44:427-30.

2. Baltov A, Mihail R, Dian E. Complications after interlocking intramedullary nailing of humeral shaft fractures. Injury 2014; 45(Suppl 1):S9-S15.

3. Korompilias AV, Lykissas MG, Kostas-Agnantis IP, Vekris MD, Soucacos PN, Beris AE. Approach to radial nerve palsy caused by humerus shaft fracture: Is primary exploration necessary? Injury 2013; 44:323-6.

4. Zhao JG, Wang J, Wang C, Kan SL. Intramedullary nail versus plate fixation for humeral shaft fractures: A systematic review of overlapping meta-analyses. Medicine 2015; 94:e599.

5. Chen F, Wang Z, Bhattacharyya T. Outcomes of nails vs. Plates for humeral shaft fractures: A medicare cohort study. J Orthop Trauma 2013; 27:68-72.

6. Vidovic D, Matejcic A, Ivica M, Jurišic D, Elabjer E, Bakota B. Minimally-invasive plate osteosynthesis in distal tibial fractures: Results and complications. Injury 2015; 46:S96-S9.

7. Metsemakers WJ, Handojo K, Reynders P, Sermon A, Vanderschot P, Nijs S. Individual risk factors for deep infection and compromised fracture healing after intramedullary nailing of tibial shaft fractures: A single centre experience of 480 patients. Injury 2015; 46:740-5.

8. Bong MR, Kummer FJ, Koval KJ, Egol KA. Intramedullary nailing of the lower extremity: Biomechanics and biology. J Am Acad Orthop Surg 2007; 15:97.

9. Polat A, Kose O, Canbora K, Yanik S, Guler F. Intramedullary nailing versus minimally invasive plate osteosynthesis for distal extra-articular tibial fractures: A prospective randomized clinical trial. J Orthop Sci 2015; 20:695-701.

10. Kejian L, Lei W, Dasheng L, Zhiwen C. Minimally invasive plating osteosynthesis for mid-distal third humeral shaft fractures. Orthopedics 2013; 36:E1025-E32.
11. Schratz W, Wörsdörfer O, Klöckner C, Götze C. Intramedullary nailing of humeral shaft fractures. Intl Orthopaedics 2002; 26:211-3.

12. Saengsin J, Vaseenon T, Pattamapaspong N, Kritsaneephaiboon A. Effectiveness of sonography assisted minimal invasive plate osteosynthesis (MIPO) compare with fluoroscope assisted in femoral shaft fracture: A cadaveric study. Injury 2017; 48:8.

13. Zhang T, Chen W, Sun J, Zhang Q, Zhang Y. Minimally invasive plate osteosynthesis technique for displaced midshaft clavicular fracture using the clavicle reductor. Intl Orthopaedics 2017; 41:1-5.

14. Yamamoto N, Ogawa K, Terada C, Okazaki Y, Munetomo K, Noda $\mathrm{T}$, et al. Minimally invasive plate osteosynthesis using posterolateral approach for distal tibial and tibial shaft fractures. Injury 2016; 47:1862-6.

15. Yang $\mathrm{KH}$, Won $\mathrm{Y}$, Kang $\mathrm{DH}$, Oh JC, Kim SJ. Role of appositional screw fixation in minimally invasive plate osteosynthesis for distal tibial fracture. J Orthop Trauma 2015; 29: 527-33.

16. Vallier HA, Cureton BA, Patterson BM. Randomized, prospective comparison of plate versus intramedullary nail fixation for distal tibia shaft fractures. J Orthop Trauma 2011; 25:736-41.

17. Vinzenz S, Alexander I, Rene EIA, Juergen O, Dietmar K, Franz K. Elastic stable intramedullary nailing is best for midshaft clavicular fractures without comminution: Results in 60 patients. Injury 2011; 42:324-9.

18. Jia-Guo Z, Jia W, Chen W, Shi-Lian K. Intramedullary nail versus plate fixation for humeral shaft fractures: A systematic review of overlapping meta-analyses. Medicine 2015; 94:e599.

19. Davies G, Yeo G, Meta M, Miller D, Hohmann E, Tetsworth K. Case-match controlled comparison of minimally invasive plate osteosynthesis and intramedullary nailing for the stabilization of humeral shaft fractures. J Orthop Trauma 2016; 30:612-7.

20. Kulkarni VS, Kulkarni MS, Kulkarni GS, Goyal V, Kulkarni MG. Comparison between antegrade intramedullary nailing (IMN), open reduction plate osteosynthesis (ORPO) and minimally invasive plate osteosynthesis (MIPO) in treatment of humerus diaphyseal fractures. Injury 2017; 48(Suppl 2):S8. 